\title{
Influence of vibration of infrared camera on the results of the analysis of thermal images
}

by M. Fidali*

\author{
*Silesian Univ. of Technology, Konarskiego 18a Str., 44-100, Gliwice, Poland, marek.fidali@polsl.pl
}

\begin{abstract}
Industrial applications of infrared cameras for continuous condition monitoring still grow. Very often in the place of camera installation the vibrations can be present. The vibrations have a negative impact on the camera operation. In the article a research and discussion connected to influence of vibration on selected features of infrared images was presented. During the research series of experimental tests in laboratory conditions were conducted and sequences of infrared images were acquired. On the basis of analysis of temperature signals a selected parameters were calculated. Values of parameters especially signals variation shown that the camera vibrations caused increasing of noise in infrared images and in some specific cases have significant influence on results of infrared image analysis.
\end{abstract}

\section{Introduction}

During the observation and recording of thermal images the infrared camera is subjected to vibration. In the case of manual inspection vibration source is a camera operator. When infrared camera is operating in on-line mode and is part of condition monitoring system of machines and processes [1], the source of vibrations acting on the camera can be machinery and equipment located in the surroundings. The vibrations with amplitudes exceeding the limit values according to camera specifications may have a negative impact on the internal structure of the device causing in the long time period malfunction of the mechatronic components of the camera (e.g. shutter mechanism, detector). The vibrations can also affect electronic systems leading to the occurrence of cold solder joint and/or loosen joints of electric connectors. The design of the camera is done in such a way as to withstand vibration. Infrared camera specifications often inform about resistance of camera mechanisms to vibration. Typically, an acceptable level of vibration is $2 \mathrm{~g}$ (acc. To IEC 68-2-6 [2]) and the maximum acceleration of the impact which camera may be exposed should not exceed $25 \mathrm{~g}$ (acc. To IEC 68-2-29 [3]). Another effect of vibration acting on the infrared camera working on-line may be the deterioration of image quality (e.g. blurring) and increase the level of disruption in diagnostic signals determined using algorithms of infrared image analysis. The article presents results of the studies in which assessed the effects of vibration on selected features of infrared images.

\section{Experimental setup}

During the studies assessed the effect of vibrations on selected features of infrared images. For this purpose test bench was prepared (Fig. 1). The test bench equipped with electrodynamic shaker (1) with power supply (2) on which an infrared camera was mounted (3). Vibration was measured with attached to the camera a piezoelectric accelerometer (4) connected to a signal analyzer (5). Signal analyzer also allowed generating control signal for shaker. IR camera and signal analyser were connected to the measuring computer (6). Object observed by a camera was reference heat source developed for the needs of the research (7). Reference source of heat contained a number of heating elements made of resistance wire and the Peltier cells, allowing to acquire the infrared images containing objects of different shapes (points, lines, planes) and positions (Fig. 2).

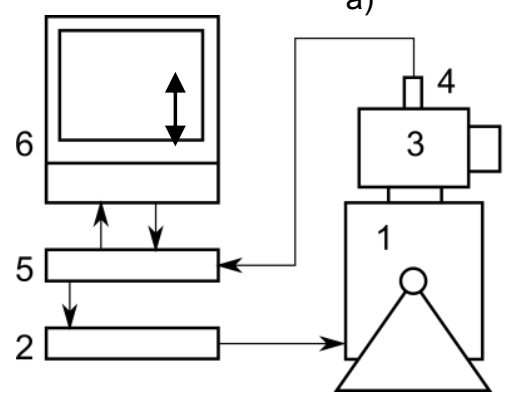

b)

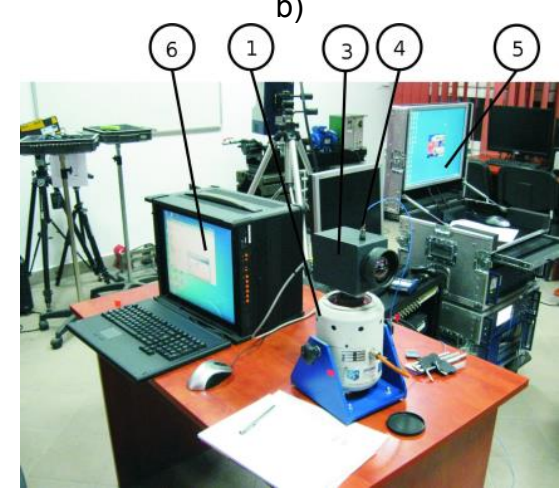

Fig. 1. Diagram (a) and real view (b) of the test bench for assessment of vibrations influence 


\subsection{1/qirt.2016.032}

During the tests the camera was subjected to vibration with two different frequencies 10 and $20 \mathrm{~Hz}$ and accelerations 1 and $5 \mathrm{~mm} / \mathrm{s}^{2}$ what corresponds to displacement respectively 0.3 and $1.3 \mu \mathrm{m}$. The camera recorded the images of the reference heat source with a frequency of $50 \mathrm{~Hz}$. Frequency of excitation and acquisition was chosen in such a way to avoid aliasing effect. For comparison purposes also ir images of reference source without exited vibrations were recorded.

\section{Measured parameters}

In order to assess vibration influence a set of 20 different measurement definitions were set in infrared images to measure temperature value as a function of time. Localisations of the measurement definitions are visible in figure 2. Used 4 measurement points (P5, P6, P10, P20), 2 measurement areas (R9, C15) and 14 measurement lines (L1, L2, L3, L4, L7, L8, L11, L12, L14, L13, L16, L17, L18, L19). In measurement points measured temporal temperature values, in area and line definitions measured $\max , \min$ and mean temperature values. Recorded images sequences allowed to obtain temperature signals in time domain which additionally transformed to frequency domain in order to generate signals spectra. Line definitions also allowed generating plots of thermal profiles as a function of time. Time functions were assessed using mean value and standard deviation parameters.

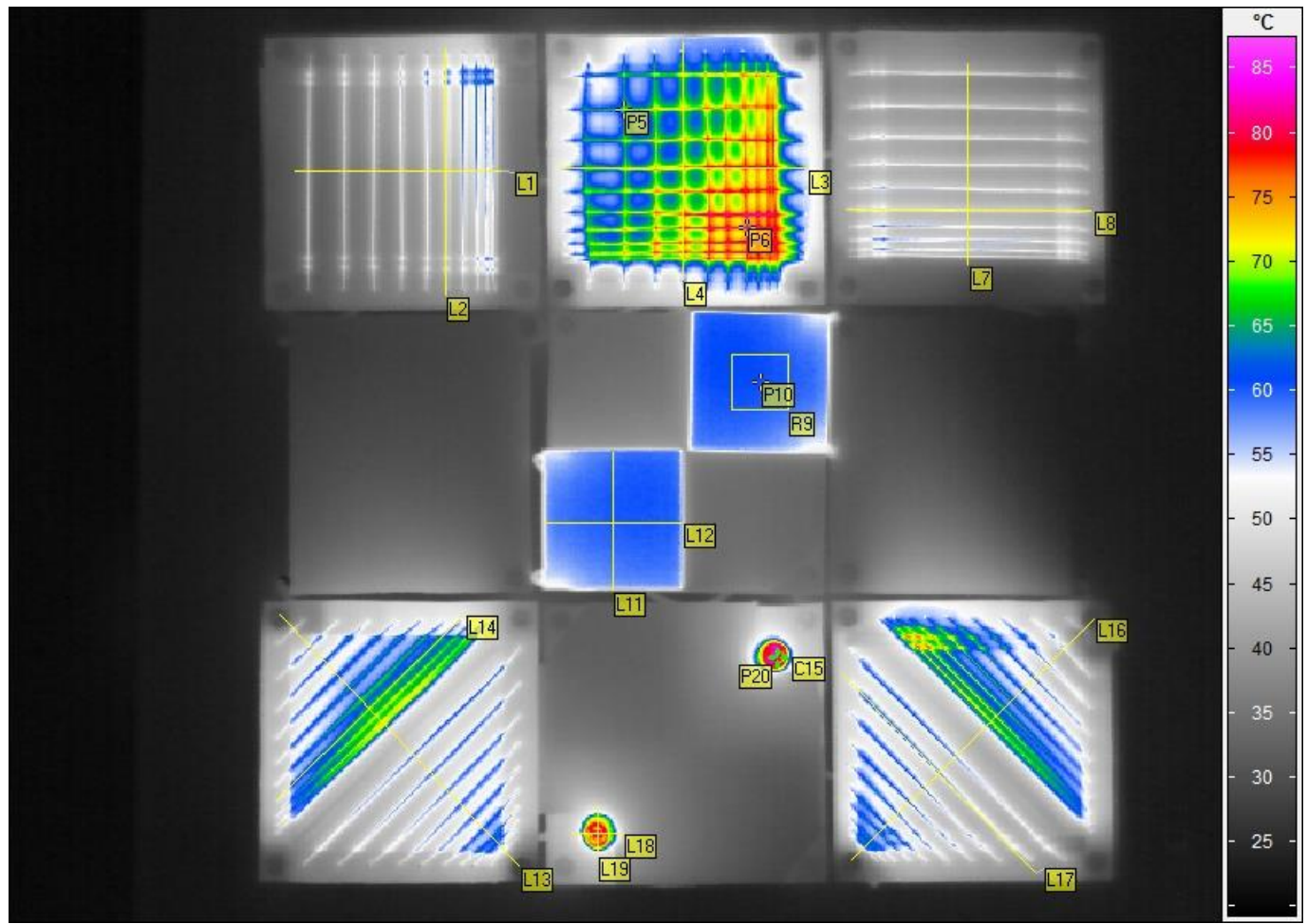

Fig. 2. Infrared image of reference heat source used during the experiments and measure definitions

\section{Results of signal analysis}

Acquired signals were estimated and compared in order to investigate influence of amplitude and frequency of camera vibrations on measured temperature values in defined measure definitions. In Fig. 3a plots of temperature signals measured in point P5 for the case when the camera has not been subjected to vibration (black dotted line) and when has vibrated with amplitude $1 \mathrm{~mm} / \mathrm{s}^{2}$ and frequency $10 \mathrm{~Hz}$ were shown. Point P5 defined at the intersection of two resistive wires. It is clearly visible influence of camera vibrations on temperature measurements what confirms spectrum of temperature signal (Fig. 3b) with visible spectral lines at frequency $10 \mathrm{~Hz}$. Presented example shows specific case associated with effect of temperature measurement at the point located on the object edge that separates the areas of two different temperatures. To avoid influence of measurements on edge investigated also how vary temperature of object with uniform temperature distribution. Such case was simulated on surface of Peltier cell where point P10 was defined. Fig. 4 shows time and frequency representation of signal measured in point $\mathrm{P} 10$ under vibration with amplitude $1 \mathrm{~mm} / \mathrm{s}^{2}$ and frequency $10 \mathrm{~Hz}$. In this case vibration component is not visible however slight increase of signal mean value is noticeable (Tab. 1). In many cases on surface of measured object exists temperature gradient and if measurements are performed close to isothermal line and ir camera vibrates, a vibration influence could also be noticeable what shown in Fig. 5 where temperature signal in time and frequency domain measured in point P20 is presented. 
a)

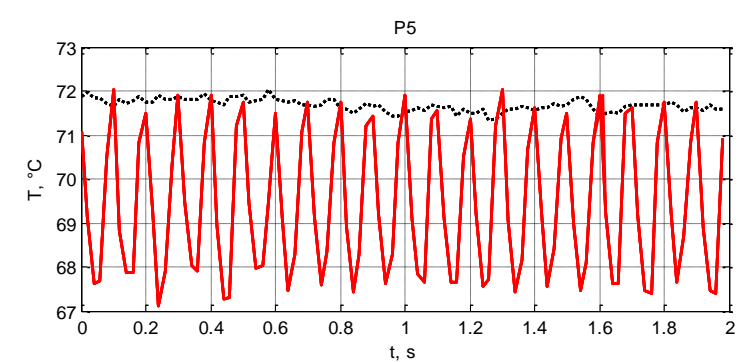

b)

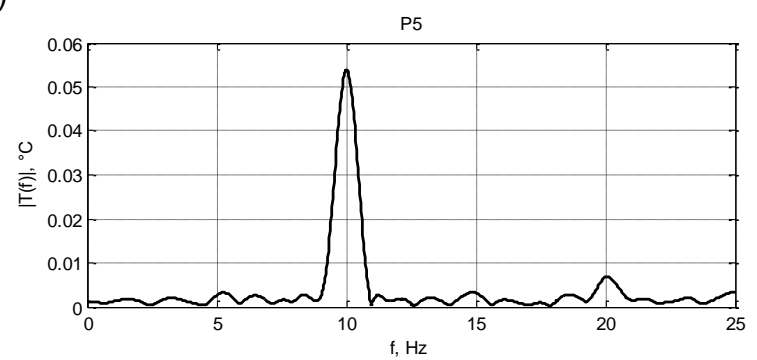

Fig. 3. Plots of temperature signals in time (black dotted line - no vibration) (a) and frequency (b) domain measured at point P5

a)

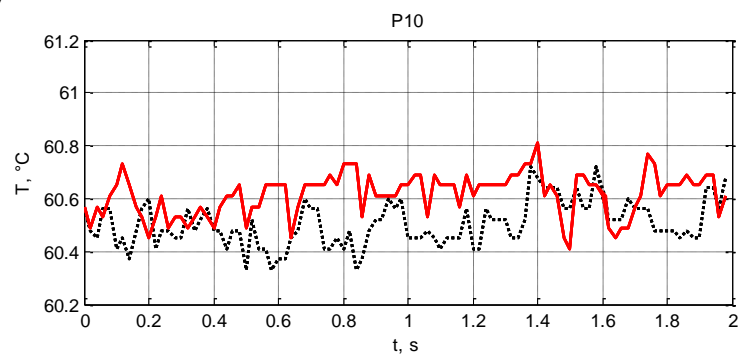

b)

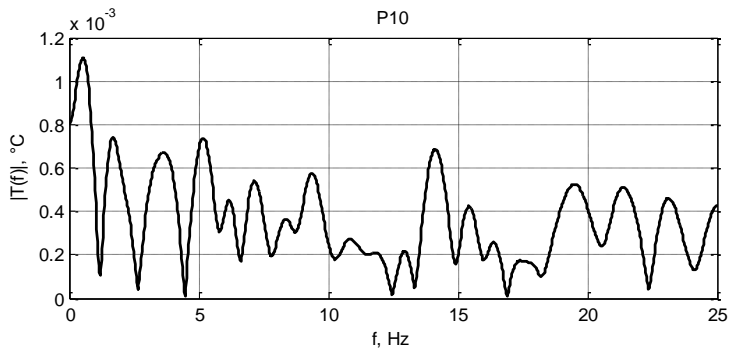

Fig. 4. Plots of temperature signals in time (black dotted line - no vibration) (a) and frequency (b) domain measured at point $P 10$

a)

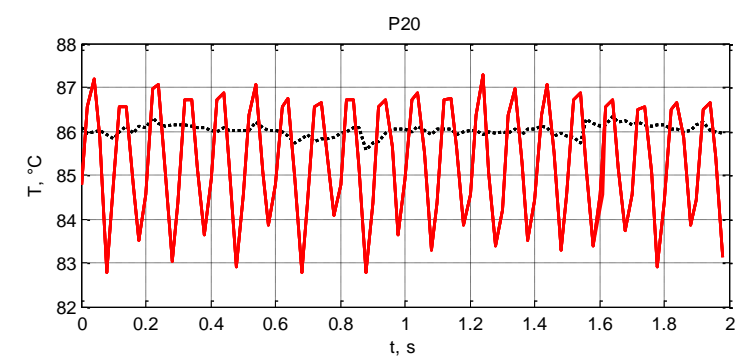

b)

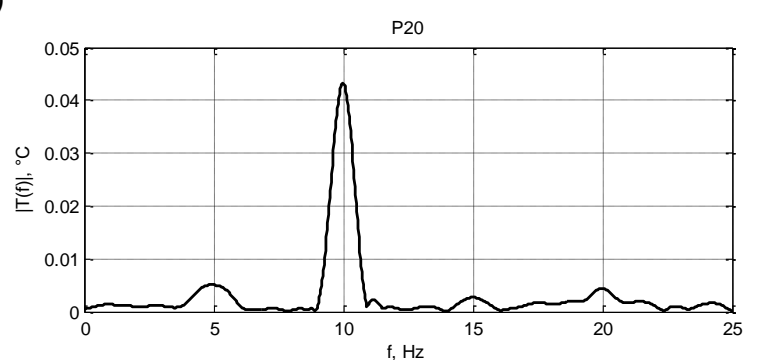

Fig. 5. Plots of temperature signals in time (black dotted line - no vibration) (a) and frequency (b) domain measured at point $P 20$

Effect of camera vibration can be observed also in the case of temperature values measured for linear profiles and areas located or intersected the edges or lines of no uniform temperature distribution. In Fig. 6 plots of linear temperature profiles over time for cases without and with camera vibration are presented. Lines L7 and L8 intersect edges of temperature gradients. The influence of vibration is clearly visible if we compare figures $6 \mathrm{c}$ and $6 \mathrm{~d}$ with figures $6 \mathrm{a}$ and $6 \mathrm{~b}$ where camera was not subjected to vibration. In case of Fig. 6e-6h line L11 and L12 was defined across uniformly heated surface of Peltier cell and in this case effect of vibration is only visible in place where profile line crosses cell edge (Fig. 6g).

In order to assess in quantitative way influence of vibration a mean value and a standard deviation of thermal signals were calculated. Signals parameters for selected measurement definitions were presented respectively in Tab. 1. and Tab. 2. Measurement definitions concern points and areas defined over non-uniformly (P5 and C15) and uniformly (P10, R9) heated areas. On the basis of presented values it is possible to compare how temperature value is changing if camera is subjected to vibration with different frequencies and amplitudes. For comparison purposes a differences between values in selected measurement definitions were calculated and presented in graphical way in Fig. 7 - Fig. 9. Differences between respectively mean values and standard deviation values of temperature signals recorded when ir camera was not subjected to vibration and excited with frequencies $10 \mathrm{~Hz}$ and $20 \mathrm{~Hz}$ were presented in Fig. 7 . In both cases vibration causes change in observed temperatures. Changes are proportional to non-uniformity of temperature distribution over measurement definition. In case of uniformly distributed temperatures the presence of vibration has a negligible impact on changes of the temperature signal. Increasing of vibration frequency cause decreasing of mean and standard deviation of temperature signal what can be seen after comparison of result presented in Fig. 7 and Fig. 8. In opposite to vibration frequency increasing amplitude of vibration causes increasing of mean and standard deviation values what is visible in Fig. 9. 
a)

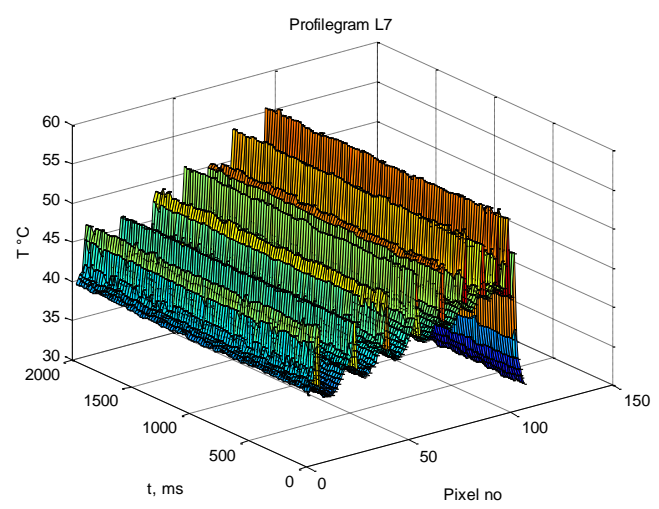

c)

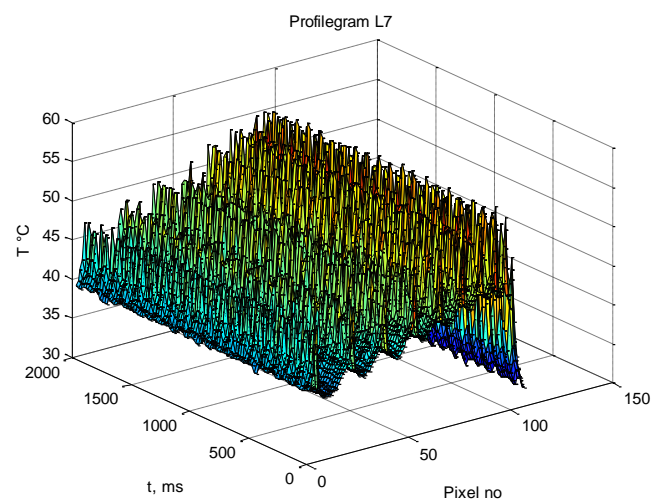

e)

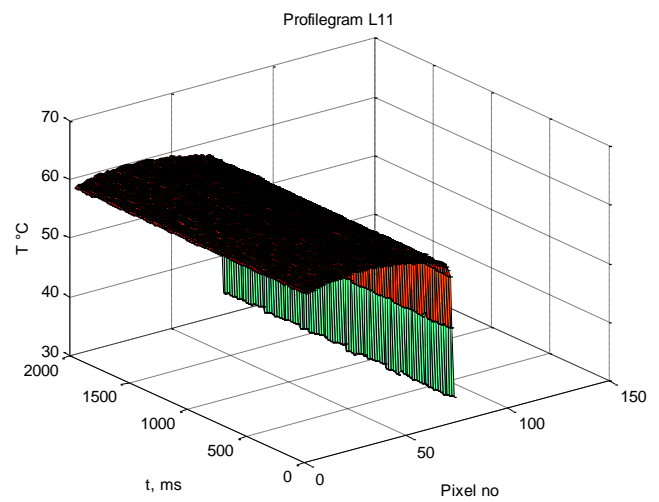

g)

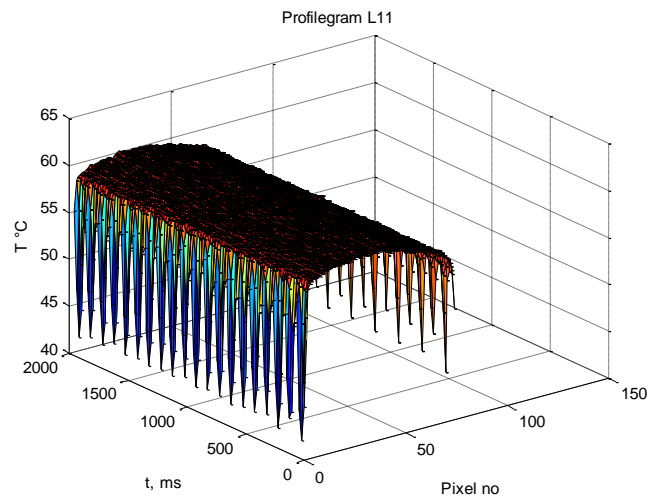

b)

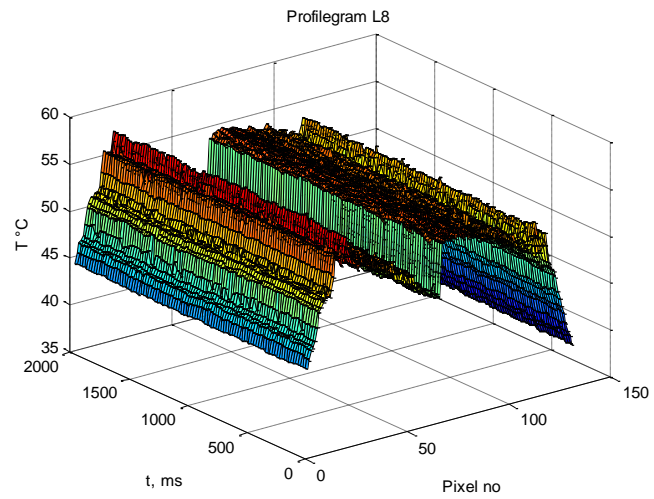

d)

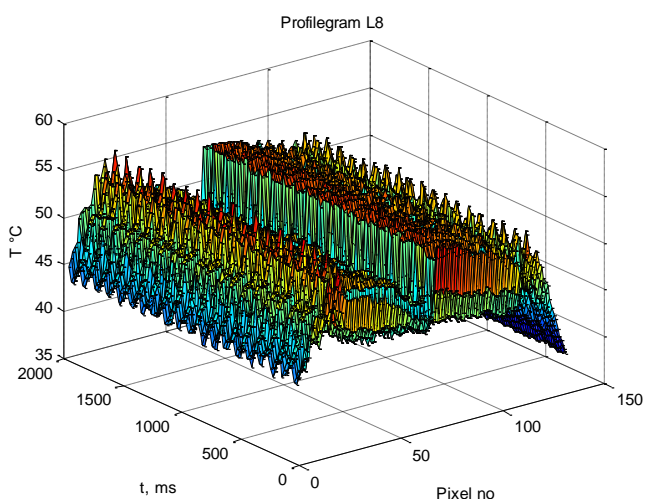

f)

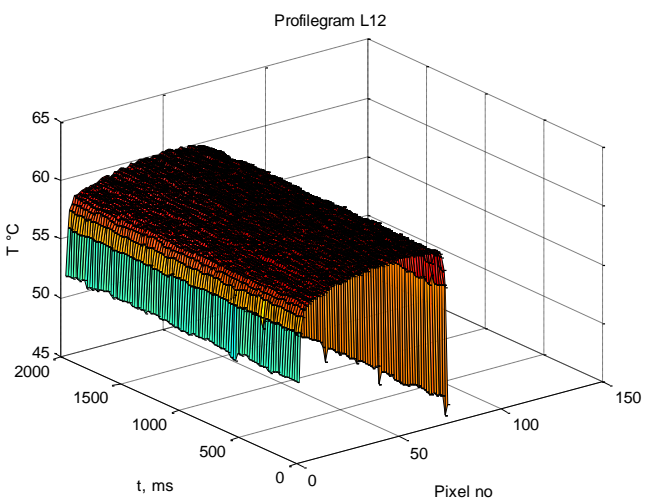

h)

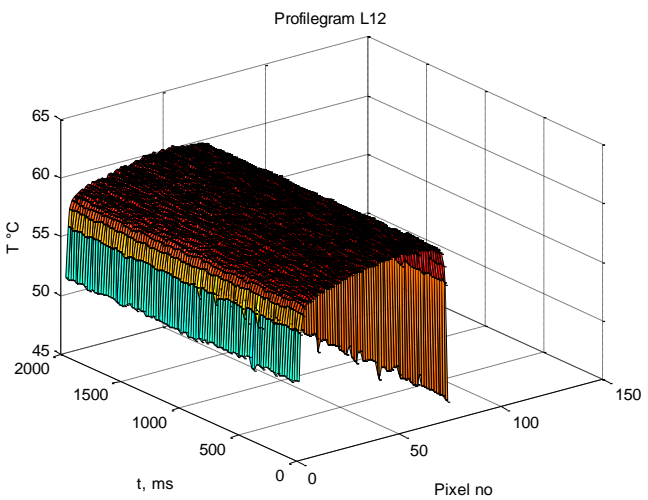

Fig. 6. Plots of temperature profiles over time for linear measurement definitions $L 7, L 8, L 11$ and $L 12$ generated for case when ir camera was not subjected to vibrations a), b), e), f) and for case when vibration was excited c), d), g), h) 
Table 1. Mean values of thermal signals at selected measurement definitions

\begin{tabular}{|l|c|c|c|c|}
\cline { 2 - 5 } \multicolumn{1}{c|}{} & \multicolumn{4}{c|}{ mean(Amplitude;Frequency) } \\
\hline Meas. Def. & $\mathrm{m}(0 ; 0)$ & $\mathrm{m}(1 ; 10)$ & $\mathrm{m}(1 ; 20)$ & $\mathrm{m}(5 ; 20)$ \\
\hline P5 & 71,68 & 69,43 & 69,39 & 68,94 \\
\hline P10 & 60,50 & 60,61 & 60,51 & 60,24 \\
\hline R9 & 60,31 & 60,34 & 60,32 & 60,11 \\
\hline C15 & 77,24 & 76,83 & 76,91 & 76,35 \\
\hline
\end{tabular}

Table 2. Standard deviation of thermal signals at selected measurement definitions

\begin{tabular}{|l|c|c|c|c|}
\cline { 2 - 5 } \multicolumn{1}{c|}{} & \multicolumn{4}{c|}{ Standard dev.(Amplitude;Frequency) } \\
\hline Meas. Def. & $\mathrm{s}(0 ; 0)$ & $\mathrm{s}(1 ; 10)$ & $\mathrm{s}(1 ; 20)$ & $\mathrm{s}(5 ; 20)$ \\
\hline P5 & 0,14 & 1,63 & 0,60 & 1,36 \\
\hline P10 & 0,08 & 0,08 & 0,09 & 0,10 \\
\hline R9 & 0,03 & 0,03 & 0,02 & 0,03 \\
\hline C15 & 0,06 & 0,26 & 0,04 & 0,15 \\
\hline
\end{tabular}

a)

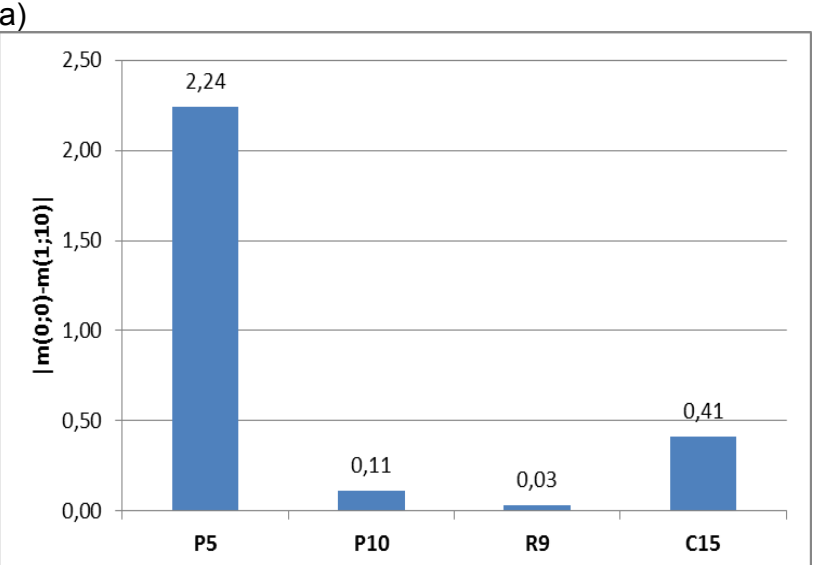

c)

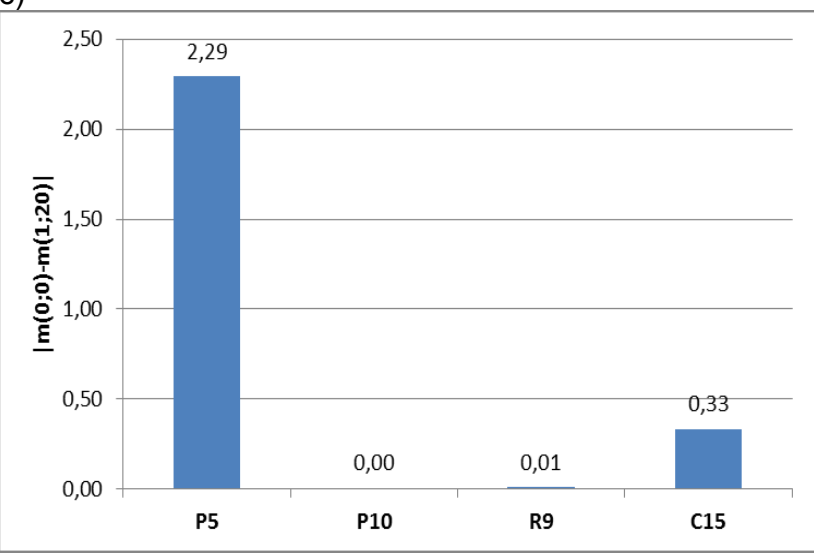

b)

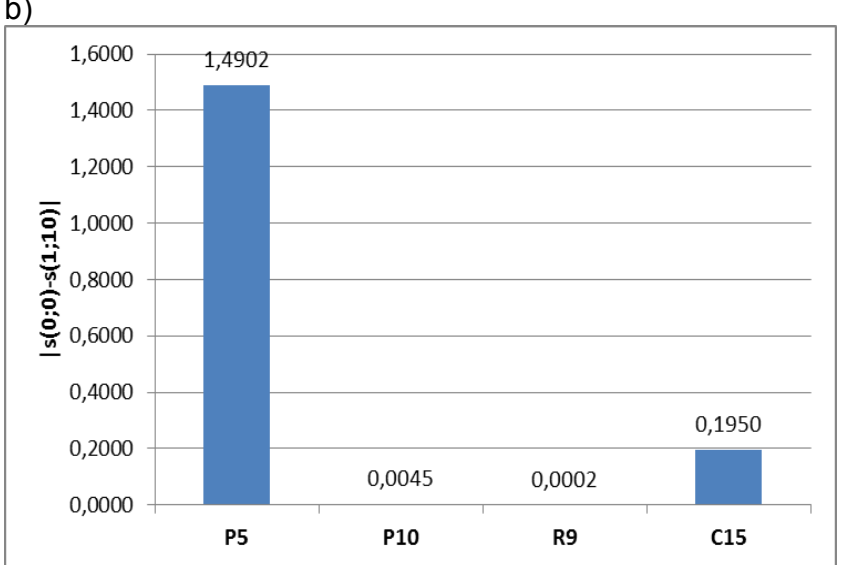

d)

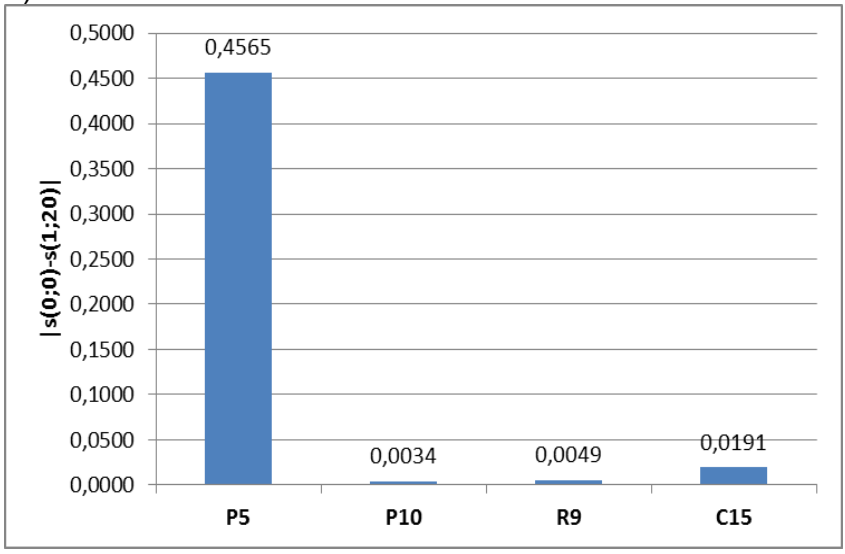

Fig. 7. Plots of mean temperature a), c) and standard deviation b), d) differences in measurement definition between values calculated when ir camera was not vibrated and vibrated with frequencies $10 \mathrm{~Hz}$ a), b) and $20 \mathrm{~Hz}$ ), d) at constant vibration amplitude $1 \mathrm{~mm} / \mathrm{s}^{2}$ 
a)

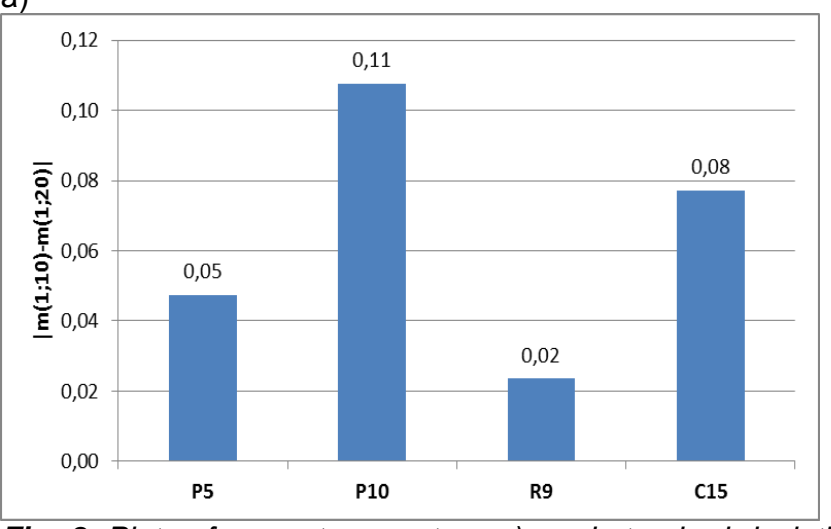

b)

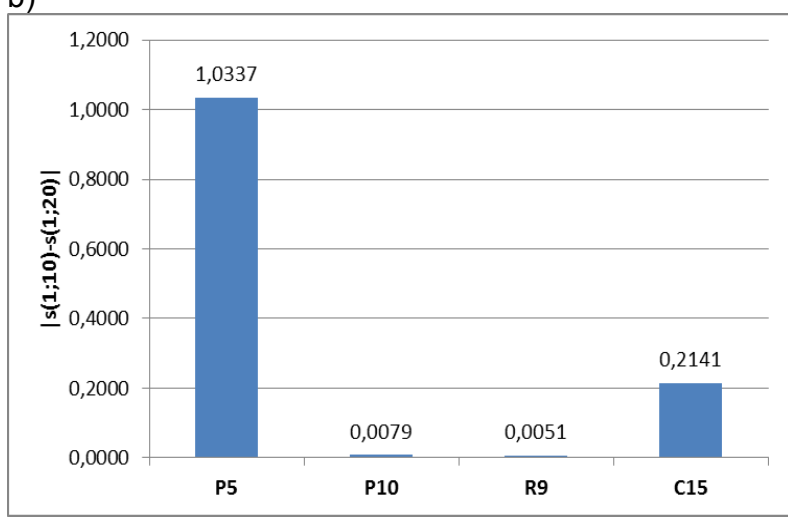

Fig. 8. Plots of mean temperature a), and standard deviation b) differences in measurement definition between values calculated when ir camera was vibrated with frequencies $10 \mathrm{~Hz}$ a) and $20 \mathrm{~Hz}$ b) at constant amplitude $1 \mathrm{~mm} / \mathrm{s}^{2}$

a)

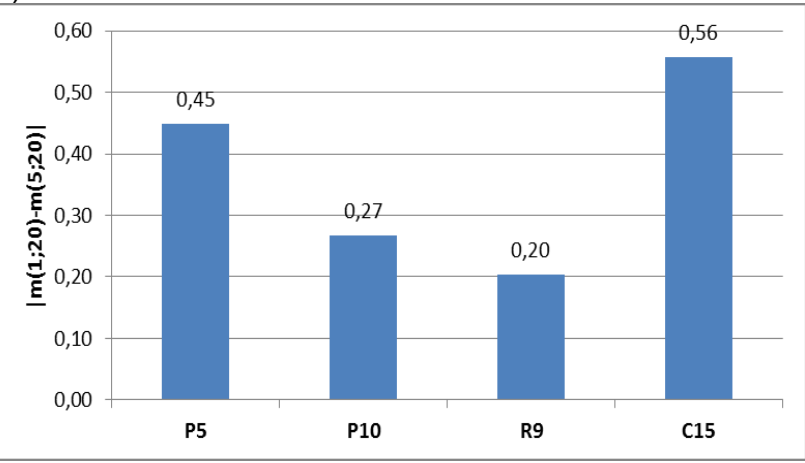

b)

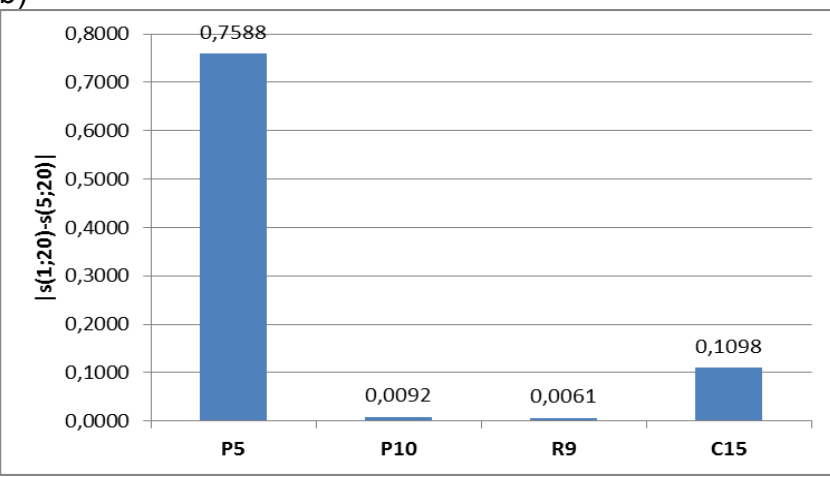

Fig. 9. Plots of mean temperature a), and standard deviation b) differences in measurement definition between values calculated when ir camera was vibrated with constant frequency $20 \mathrm{~Hz}$ and amplitudes $1 \mathrm{~mm} / \mathrm{s}^{2}$ a) and $5 \mathrm{~mm} / \mathrm{s}^{2}$

\section{Conclusions}

Studies concerning the influence of infrared camera vibration on the features of the recorded images have not been earlier carried out what confirms lack of publications on this subject. Results of the presented in this article research shown that vibrations have an adverse impact on the diagnostic signal extracted from a sequence of infrared images. The presence of high amplitude and low frequency vibrations in place of mounting infrared camera, significantly affects the increase of noise in the infrared images. Intensive vibration with low frequencies can significantly reduce the sensitivity of the camera and therefore ability to the recognition of small changes in temperature distribution of observed objects. Mean values of the temperature signals increases proportional to amplitude of camera vibrations and inversely to vibration frequency. In case of measurement definitions located on object surface with uniform temperature distribution influence of vibration signals are not clearly visible. Influence of camera vibration depends on the relationships between the parameters of the camera, detector spatial resolution, lens focal length and distance camera - object as well as the size of the observed object. Vibration impact is grater the more the camera is distanced from the observed object. In the case of the use of infrared cameras to continuous monitoring of industrial objects, it is necessary to find right place for mounting of a camera. If in camera mounting place are noticeable vibrations it is necessary to identify vibration characteristics and apply appropriate anti-vibrations mounting or change a mounting place. In some cases it is worth to consider application of camera vibrations monitoring system based for example on MEMS accelerometers. One could be expected that the observation of vibrating object using stationary camera also affect the quality of the acquired infrared image sequence. Presented research revealed that infrared camera could be used to low frequency vibration measurements what will be subject of author's further research.

\section{REFERENCES}

[1] Fidali M.: Methodology of thermographical diagnostics of technical objects (in polish). Wydawnictwo Naukowe Instytutu Technologii Eksploatacji - PIB. 2013. http://ipkm.polsl.pl/index.php?n=KsiazkilPKM.HomePage

[2] IEC 68-2-6, Environmental testing -Part 2: Tests -Test Fc: Vibration (sinusoidal)

[3] IEC 68-2-29, Basic environmental testing procedures - Part 2: Tests - Test Eb and guidance: Bump 